\title{
Spectroscopic mode identification for the slowly pulsating B star HD 147394^
}

\author{
M. Briquet ${ }^{1}$, C. Aerts $^{2}$, P. Mathias ${ }^{3}$, R. Scuflaire ${ }^{1}$, and A. Noels ${ }^{1}$ \\ ${ }^{1}$ Institut d'Astrophysique et de Géophysique de Liège, Université de Liège, allée du Six Août 17, 4000 Liège, Belgium \\ ${ }^{2}$ Instituut voor Sterrenkunde, Katholieke Universiteit Leuven, Celestijnenlaan 200 B, 3001 Leuven, Belgium \\ 3 Observatoire de la Côte d'Azur, Département Fresnel, UMR 6528, 06304 Nice Cedex 4, France
}

Received 27 November 2002 / Accepted 15 January 2003

\begin{abstract}
We present ground-based high-resolution high $S / N$ spectra of the slowly pulsating B star HD 147394 . Two frequencies are clearly found in the moments of the Si II 4128-4130 doublet: $f_{1}=0.8008 \mathrm{c} \mathrm{d}^{-1}$ and $f_{2}=0.7813 \mathrm{~cd}^{-1}$. The frequency $f_{1}$ is also found in the HIPPARCOS data. A third frequency is also present in our spectroscopic data: $f_{3}=0.7175 \mathrm{c} \mathrm{d}^{-1}$ or its aliases due to the time sampling. A mode identification is performed by means of a new version of the moment method optimized for multiperiodic signals. It points towards several equivalent solutions of non-axisymmetric $\ell \leq 3$ for all three modes. We have too limited information to perform seismic modelling at this stage, but we do show that the different possibilities for the mode identifications are compatible with pulsational models for SPBs.
\end{abstract}

Key words. line: profiles - stars: early-type - stars: oscillations - stars: rotation - stars: variables: general stars: individual: HD 147394

\section{Introduction}

The slowly pulsating B stars (SPBs) were introduced as a new class of pulsating B-type stars by Waelkens (1991). They are situated along the main-sequence with spectral types ranging from B2 to B9 and masses ranging from $3 M_{\odot}$ to $7 M_{\odot}$. They show light and line-profile variations, that are multiperiodic with periods of the order of days. This variability is understood in terms of non-radial stellar pulsations and their oscillation modes are high-order g-modes. Theoretical models attribute the pulsational nature of SPBs to the $\kappa$-mechanism, acting in the metal opacity bump at $2 \times 10^{5} \mathrm{~K}$.

The main future goal of the study of SPBs is to perform asteroseismology, i.e. to probe their internal structure by using their observed pulsational characteristics. To do this, many pulsation frequencies and modes must be detected, which is an observational challenge because of the long pulsation periods of SPBs.

From the HIPPARCOS mission, a huge number of B-type stars have been classified as new SPBs by Waelkens et al. (1998). These new SPBs almost fully cover the theoretical instability domain calculated for such stars by Pamyatnykh (1999). A sample of about 20 stars among these SPBs has been selected for long-term photometric and spectroscopic monitoring with the aim of providing an inventory of the

Send offprint requests to: M. Briquet,

e-mail: M.Briquet@ulg.ac.be

* Based on observations obtained with the spectrograph Aurélie attached to the 1.52-m telescope of the Observatoire de Haute-Provence, France. observational characteristics of the pulsations (Aerts et al. 1999; De Cat et al. 2000; De Cat 2001; Mathias et al. 2001; De Cat \& Aerts 2002). We refer to De Cat (2002) for an observational overview.

This paper is devoted to the analysis of one of these stars: HD 147394. It is the SPB for which most high-resolution spectra are available thus far. We performed a frequency analysis on these data, followed by a mode identification from the line-profile variations. The plan of the paper is the following. In Sect. 2 we give a description of our data and we derive some physical parameters of HD 147394. The results of the frequency analysis from derived quantities based upon the spectroscopic observations are described in Sect. 3. In Sect. 4 we identify the modes of HD 147394 by means of a new version of the moment method (Briquet \& Aerts 2003). As this is one of the first spectroscopic mode identifications ever done for an SPB, we compare our identification results with theoretical pulsation models as a compatibility check in Sect. 5. Finally, we give a summary in Sect. 6 .

\section{The spectroscopic data and the physical parameters of HD 147394}

We selected HD 147394 among the many SPBs discovered from the HIPPARCOS mission (Waelkens et al. 1998) so that we have at our disposal HIPPARCOS photometry, which clearly reveals the frequency $0.80027 \mathrm{~cd}^{-1}$ (Mathias et al. 2001). We note that this dataset shows evidence of multiperiodicity but a value for a second frequency is not clear. 


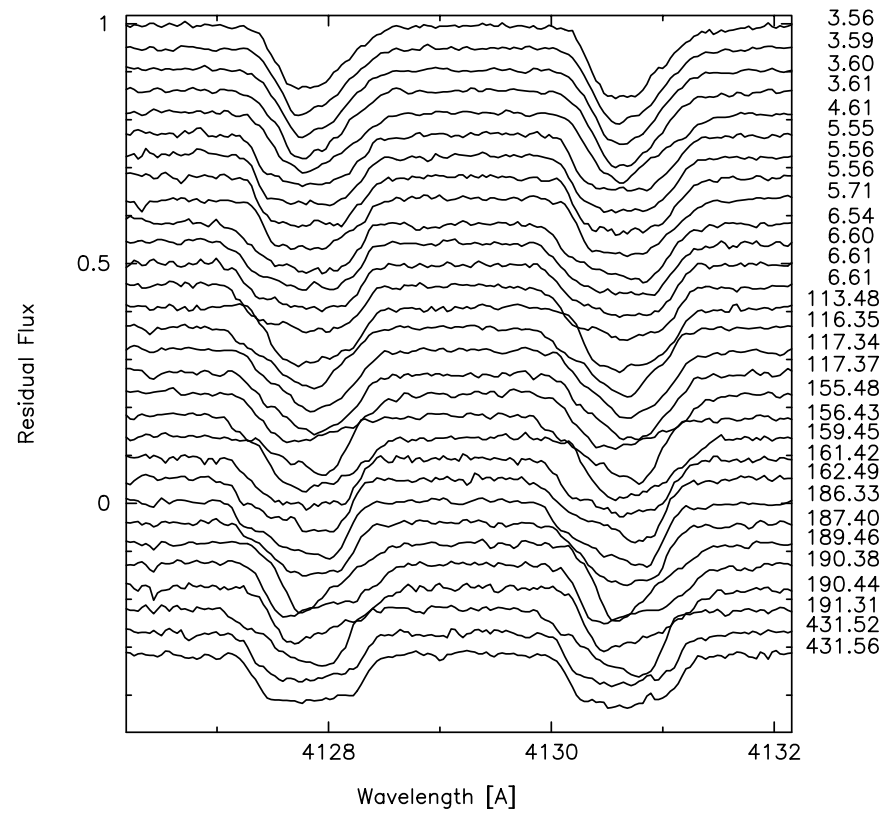

Fig. 1. Line-profile variations of the Si II 4128-4130 doublet. Observation dates are indicated on the right of the panel (+2450850 HJD).

Table 1. Observing logbook of our spectroscopy of HD 147394.

\begin{tabular}{|c|c|c|}
\hline \multirow[t]{2}{*}{$\begin{array}{l}\text { Number of } \\
\text { observations }\end{array}$} & \multicolumn{2}{|c|}{$\begin{array}{c}\text { JD } \\
2450850+\end{array}$} \\
\hline & Start & End \\
\hline 47 & 1 & 6 \\
\hline 14 & 113 & 117 \\
\hline 14 & 155 & 162 \\
\hline 13 & 186 & 192 \\
\hline 117 & 431 & 437 \\
\hline 45 & 457 & 461 \\
\hline
\end{tabular}

Line-profile variations have already been reported by Masuda \& Hirata (2000), who gathered 30 spectra in 5 nights. We have a much more extensive dataset which consists in 250 useful spectra obtained with the spectrograph Aurélie at OHP during 6 separate weeks of monitoring spread over 460 days. The number of observations and the ranges of their Julian Dates are given in Table 1. The spectral domain is limited and was chosen in order to get the SiII-doublet with lines at $\lambda \lambda 4128,4130 \AA$. The signal-to-noise ratio is about 200 . For a complete description of the observations and data reductions we refer to Mathias et al. (2001). Figure 1 represents several of the observed line-profile variations.

We also have a few Geneva data points at our disposal of the star from which we derive the stellar parameters, as the spectra have only very small spectral coverage. The effective temperature and the gravity of HD 147394 are obtained by means of the photometric calibration by Künzli et al. (1997) to the mean magnitudes in the Geneva filters. The distance, derived from the parallax measured by HIPPARCOS, and the average visual magnitude, give the absolute visual magnitude. Taking into account the bolometric correction (BC), which is

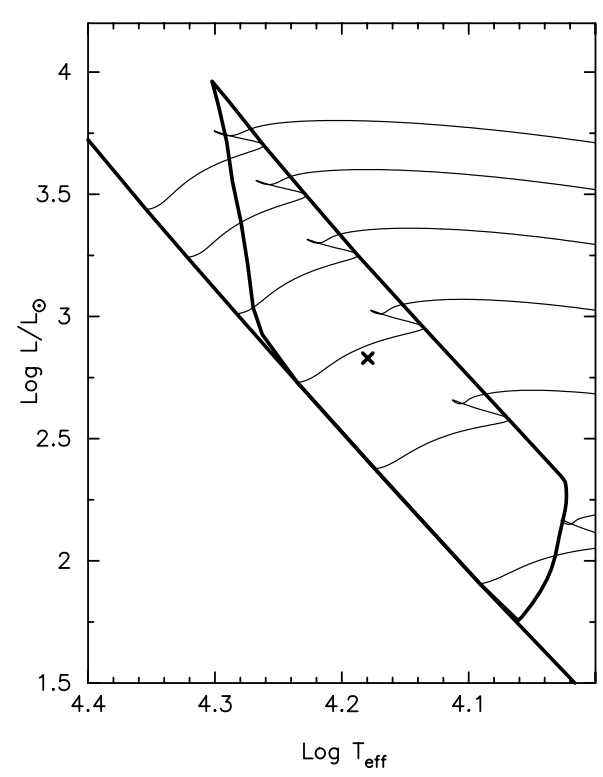

Fig. 2. Position of HD 147394 in the HR diagram. The theoretical SPB instability strip is calculated by Pamyatnykh (1999). The star is situated on the evolutionary track published by Schaller et al. (1992) corresponding to some $5 M_{\odot}$.

calculated by means of Flower's relation (1996) between $T_{\text {eff }}$ and $\mathrm{BC}$, one obtains the bolometric magnitude and the luminosity. With the values for the effective temperature and the luminosity one estimates the mass, e.g. by using the evolutionary tracks published by Schaller et al. (1992). We also calculated the radius. The results are the following:

$$
\left\{\begin{array}{l}
\log T_{\text {eff }}=4.17 \pm 0.01 \\
\log g=4.00 \pm 0.15 \\
\log L / L_{\odot}=2.80 \pm 0.15 \\
M=5.0 \pm 0.5 M_{\odot} \\
R=3.7 \pm 0.8 R_{\odot}
\end{array}\right.
$$

Because we have only a few Geneva data, we checked our result with those available in the literature and derived from spectroscopic data. In Smith (1997), we found $\log T_{\text {eff }}=4.18$ and $\log g=3.93$, which is in agreement with our estimates based on the Geneva photometry. Smith \& Dworetsky (1993) have found the star to have solar iron abundance $(\log N(\mathrm{Fe})=7.65 \pm 0.15)$.

The position of this B5 IV star in the theoretical HR diagram falls in the centre of the SPB instability strip (see Fig. 2).

\section{Frequency analysis}

We extracted the measurements of the doublet Si II centered at $\lambda \lambda$ 4128,4130 ̊. From these spectral lines we computed the first three velocity moments $\langle v\rangle,\left\langle v^{2}\right\rangle$ and $\left\langle v^{3}\right\rangle$ (see Aerts et al. 1992 for a definition of the moments of a line profile) with the aim of performing a frequency analysis. We used the PDM method (Stellingwerf 1978), Scargle method (Scargle 1981) and the CLEAN method (Roberts et al. 1987). We tested frequencies from 0 to 3 cycles per day $\left(\mathrm{c} \mathrm{d}^{-1}\right)$ with a frequency 

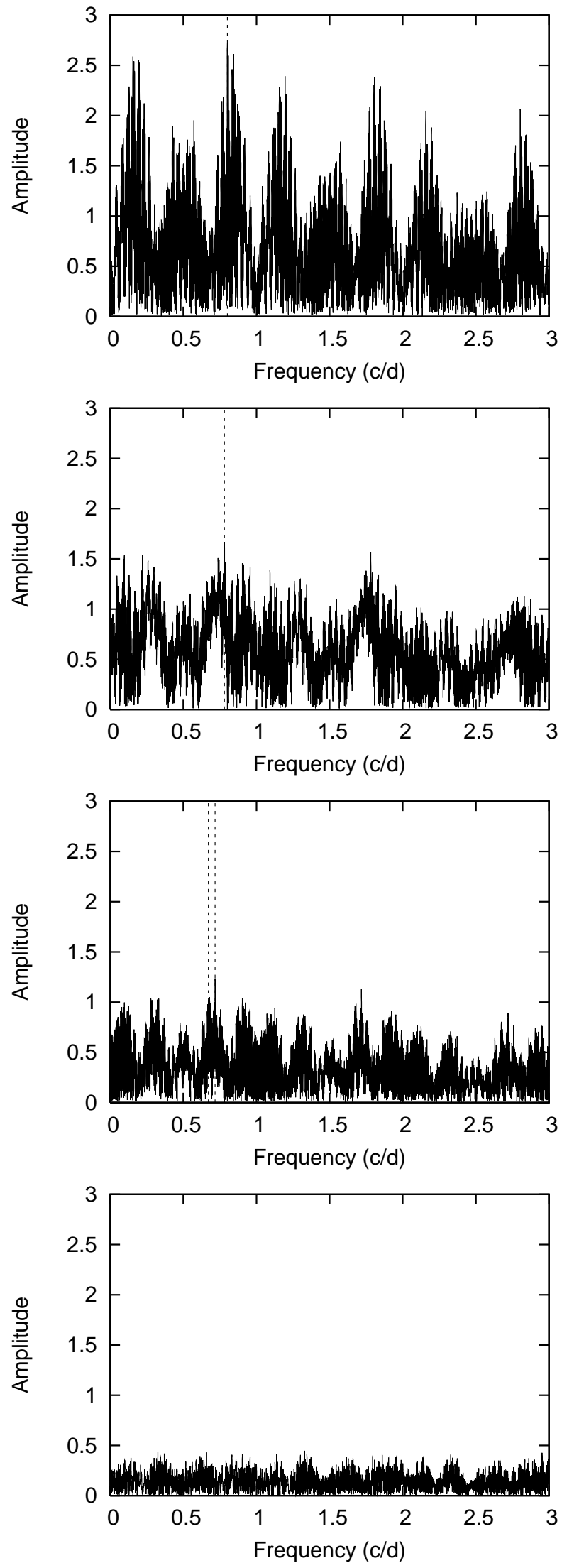

Fig. 3. From top to bottom: Scargle periodograms of the radial velocity derived from the Si II 4130 line, of this data prewhitened with $f_{1}$, of this data prewhitened with $f_{1}$ and $f_{2}$, of this data prewhitened with $f_{1}$, $f_{2}$ and $f_{3}$. The $4 S / N$ level is situated at $0.74 \mathrm{~km} \mathrm{~s}^{-1}$ in the third panel.
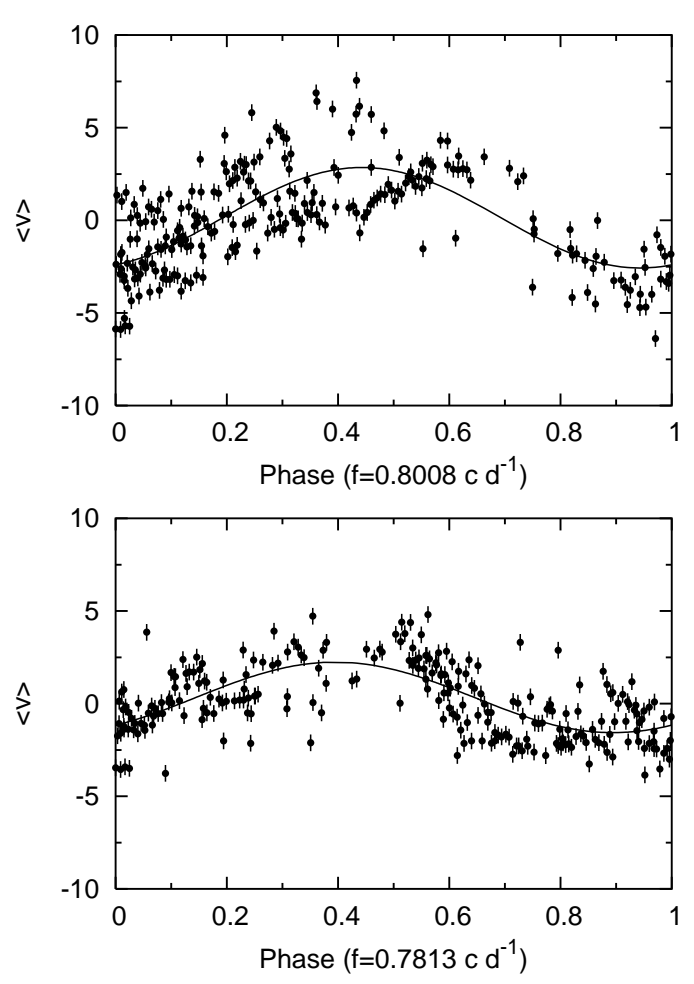

Fig. 4. Upper panel: phase diagram of the radial velocity computed from the Si II 4130 line for $f_{1}=0.8008 \mathrm{c} \mathrm{d}^{-1}$. Lower panel: phase diagram of the data prewhitened with $f_{1}$ for $f_{2}=0.7813 \mathrm{~cd}^{-1}$.

step of $0.0001 \mathrm{c} \mathrm{d}^{-1}$. The error estimate of our determined frequencies is between $0.0001 \mathrm{c} \mathrm{d}^{-1}$ and $0.001 \mathrm{c} \mathrm{d}^{-1}$. We obtained the same results with the three methods and for both Si lines.

In $\langle v\rangle,\left\langle v^{2}>\right.$ and $\left\langle v^{3}>\right.$, we found the frequency $f_{1}=0.8008 \mathrm{c} \mathrm{d}^{-1}$, which corresponds to the frequency found in the HIPPARCOS data. After prewhitening of the data with this dominant frequency, we obtained a second frequency clearly present in $\langle v\rangle$ and $\left\langle v^{3}\right\rangle$, which is $f_{2}=0.7813 \mathrm{c} \mathrm{d}^{-1}$. These two frequencies reduce the standard deviation of the first moment by $50 \%$. Note that a fit for $1+f_{2}$ is slightly less good. $f_{1}$ and $1+f_{2}$ reduce the standard deviation by $48 \%$. A fit with $f_{1}$ and $1+f_{2}$ leads to a smaller amplitude for the second mode compared to a fit with $f_{1}$ and $f_{2}: 1.70 \mathrm{~km} \mathrm{~s}^{-1}$ for $1+f_{2}$ instead of $2.05 \mathrm{~km} \mathrm{~s}^{-1}$ for $f_{2}$. We then kept $f_{2}$ for the second frequency. Scargle periodograms are shown in Fig. 3 and phase diagrams of the first moment for the Si II 4130 line are shown in Fig. 4.

After prewhitening of the data with $f_{1}$ and $f_{2}$, the residuals show evidence of a third frequency. Two frequencies are apparent: $f_{3}=0.7175 \mathrm{c} \mathrm{d}^{-1}$ or $f_{3}^{\prime}=0.6710 \mathrm{~cd}^{-1}$. The first one $\left(f_{3}\right)$ occurs after prewhitening with $f_{1}$ and $f_{2}$ (see third panel of Fig. 3) while the second one $\left(f_{3}^{\prime}\right)$ is the highest peak if we prewhiten with slightly different values for $f_{1}$ and $f_{2}$ (e.g. $0.8006 \mathrm{c} \mathrm{d}^{-1}$ and $0.7814 \mathrm{~cd}^{-1}$ ) within the error estimate. Together with $f_{1}$ and $f_{2}$, they reduce respectively $61 \%$ and $59 \%$ of the standard deviation in the first moment. Phase diagrams of the radial velocity, prewhitened with $f_{1}$ and $f_{2}$, for $f_{3}=$ $0.7175 \mathrm{c} \mathrm{d}^{-1}$ and for $f_{3}^{\prime}=0.6710 \mathrm{~cd}^{-1}$ are shown in Fig. 5 . We note that $f_{3}$ reduces the standard deviation slightly better than $f_{3}^{\prime}$. The difference between both candidate frequencies is $0.0465 \mathrm{c} \mathrm{d}^{-1}$, which corresponds to about three weeks. We also 
Table 2. Highest peaks in the window function (expressed in $\mathrm{cd}^{-1}$ ).

\begin{tabular}{llrl}
\hline \hline $\mathrm{p} 1$ & 1.0001 & $\mathrm{p} 9$ & 0.0024 \\
$\mathrm{p} 2$ & 1.0026 & $\mathrm{p} 10$ & 1.0070 \\
$\mathrm{p} 3$ & 0.0068 & $\mathrm{p} 11$ & 0.0397 \\
$\mathrm{p} 4$ & 0.9957 & $\mathrm{p} 12$ & 0.9629 \\
p5 & 0.0440 & $\mathrm{p} 13$ & 0.9561 \\
p6 & 0.0372 & $\mathrm{p} 14$ & 0.0465 \\
p7 & 1.0398 & $\mathrm{p} 15$ & 2.0027 \\
p8 & 0.0044 & & \\
\hline
\end{tabular}

Table 3. Amplitudes and phases of the least-squares sine fits to the observed first moment computed from the Si II 4130 line, together with their standard errors for $f_{1}, f_{2}$ and $f_{3}$, for $f_{1}, f_{2}$ and $f_{3}^{\prime}$.

\begin{tabular}{ccc}
\hline \hline & Amplitude $\left(\mathrm{km} \mathrm{s}^{-1}\right)$ & Phase (degrees) \\
\hline$f_{1}$ & $3.28 \pm 0.11$ & $109 \pm 2$ \\
$f_{2}$ & $2.05 \pm 0.11$ & $136 \pm 2$ \\
$f_{3}$ & $1.37 \pm 0.11$ & $60 \pm 5$ \\
\hline$f_{1}$ & $3.07 \pm 0.12$ & $108 \pm 2$ \\
$f_{2}$ & $1.84 \pm 0.12$ & $135 \pm 3$ \\
$f_{3}^{\prime}$ & $1.07 \pm 0.11$ & $152 \pm 6$ \\
\hline
\end{tabular}

notice that the time span between two missions of observations is about three weeks or about a multiple of three weeks except between the second and third missions. This indicates that the frequencies may be aliases. We computed the window function for frequencies between $0.0001 \mathrm{~cd}^{-1}$ and $10 \mathrm{~cd}^{-1}$ with a step of $0.0001 \mathrm{c} \mathrm{d}^{-1}$. The 15 highest peaks of this function are listed in Table 2. The 14th peak is exactly $0.0465 \mathrm{c} \mathrm{d}^{-1}$. One also remarks that only four frequencies of the list are independent. We conclude that $f_{3}$ and $f_{3}^{\prime}$ are aliases due to the time sampling. We also note that additional aliases of $f_{3}$ and $f_{3}^{\prime}$ cannot be excluded (see third panel of Fig. 3). In the following, we continue to work with $f_{3}$ and $f_{3}^{\prime}$, just to see if our results for the two main modes $f_{1}$ and $f_{2}$ are influenced by using different values for the frequency of the third mode.

In order to determine the significance of both aliases, we considered the $4 S / N$ criterion introduced by Breger et al. (1993). The noise is defined as the average value of the amplitude of the peaks in the surroundings of the suspected frequencies (in the interval $[0,2] \mathrm{c} \mathrm{d}^{-1}$ for this case) in an oversampled periodogram after prewhitening of the candidate frequencies. Frequencies for which the peak amplitude is higher than 4 times the noise level are retained. In Fig. 3 we show the Scargle periodogram. As the $4 S / N$ level is $0.74 \mathrm{~km} \mathrm{~s}^{-1}$, both aliases must be retained based on this criterion.

The amplitudes and phases of the least-squares sine fits to the observed first moment for the combinations $f_{1}, f_{2}, f_{3}$ and $f_{1}$, $f_{2}, f_{3}^{\prime}$ are listed in Table 3 .

\section{Mode identification with the moment method}

Among techniques of mode identification from line-profile variations, only the line-profile fitting method and the moment method derive the full pulsational information. Because, even
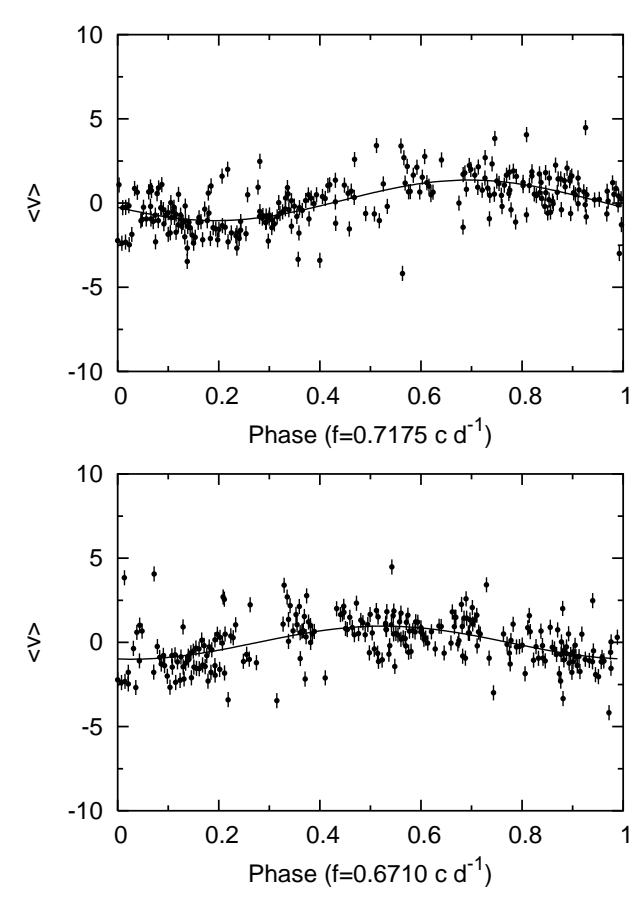

Fig. 5. Upper panel: phase diagram of the radial velocity computed from the SiII 4130 line, prewhitened with $f_{1}$ and $f_{2}$, for $f_{3}=$ $0.7175 \mathrm{~cd}^{-1}$. Lower panel: phase diagram of the data prewhitened with $f_{1}$ and $f_{2}$, for $f_{3}^{\prime}=0.6710 \mathrm{~cd}^{-1}$.

with current computers, a simultaneous identification of multiple modes is not possible by direct line-profile fitting, the moment method turns out to be very useful. With this method, the wavenumbers $(\ell, m)$ and the other continuous velocity parameters are determined in such a way that the theoretically computed first three moment variations best fit the observed ones. We refer to Briquet \& Aerts (2003) for the latest version of the technique, which was improved by these latter authors in order to perform a simultaneous identification of all the modes that are present in the data. We also point out that the method is no longer restricted to slow rotators but is extended to rotating pulsating stars by Briquet $\&$ Aerts (2003) by using the theory derived by Lee \& Saio (1987). The use of this latter formalism instead of the non-rotating one can be relevant for SPBs since their observed ratios of the rotational frequency to the pulsational frequency are in general larger than 0.1 (De Cat 2001).

Because of the alias problem for the third mode, we identified modes simultaneously for both the combination $f_{1}, f_{2}$ and $f_{3}$ and the combination $f_{1}, f_{2}$ and $f_{3}^{\prime}$ in order to check the consistency of the results. Moreover, for both cases, we performed one identification by using the non-rotating formalism and one identification by using Lee \& Saio's formalism.

To compute the theoretical moments, we took a linear limbdarkening coefficient $u$ of 0.36 (see e.g. Wade \& Rucinski 1985). To identify the modes, we covered the parameter space by varying the free parameters in the following way: the projected rotational velocity $v_{\Omega}$ from 1 to $35 \mathrm{~km} \mathrm{~s}^{-1}$ with a step $1 \mathrm{~km} \mathrm{~s}^{-1}$, the inclination of the star $i$ from $5^{\circ}$ to $90^{\circ}$ with a step $5^{\circ}$, the line-profile width from 1 to $20 \mathrm{~km} \mathrm{~s}^{-1}$ with a step $1 \mathrm{~km} \mathrm{~s}^{-1}$. We used the $K$-value given by $K=G M / \omega^{2} R^{3}$, where $M$ is the mass, $R$ the radius and $\omega$ the angular pulsation 
Table 4. The ten best solutions of the mode identification by means of the non-rotating formalism through the discriminant $\Sigma$, using the Si II $4130 \AA$ line. $A_{\mathrm{p}}$ is the amplitude of the radial part of the pulsation velocity, expressed in $\mathrm{km} \mathrm{s}^{-1} ; v_{\mathrm{r}, \max }$ and $v_{\mathrm{t}, \text { max }}$ are respectively the maximum radial and tangential surface velocity due to the three modes, expressed in $\mathrm{km} \mathrm{s}^{-1} ; i$ is the inclination angle; $v_{\Omega}$ is the projected rotational velocity, expressed in $\mathrm{km} \mathrm{s}^{-1}$ and $\sigma$ is the intrinsic line-profile width, also expressed in $\mathrm{km} \mathrm{s}^{-1}$. The second-but-last column contains the solution that occurs at position 20. The upper and lower part of the table correspond respectively to an identification for $f_{1}, f_{2}, f_{3}$ and for $f_{1}$, $f_{2}, f_{3}^{\prime}$.

\begin{tabular}{|c|c|c|c|c|c|c|c|c|c|c|c|c|c|}
\hline$\left(\ell_{1}, m_{1}\right)$ & $(1,1)$ & $(3,-2)$ & $(1,1)$ & $(3,-2)$ & $(1,1)$ & $(3,-2)$ & $(1,1)$ & $(1,1)$ & $(2,-2)$ & $(2,-2)$ & $\ldots$ & $(3,-2)$ & $\ldots$ \\
\hline$\left(\ell_{2}, m_{2}\right)$ & $(3,-1)$ & $(3,-1)$ & $(3,-1)$ & $(3,-1)$ & $(3,-1)$ & $(3,-1)$ & $(2,-1)$ & $(2,-1)$ & $(2,-1)$ & $(2,-1)$ & $\ldots$ & $(3,-1)$ & $\ldots$ \\
\hline$\left(\ell_{3}, m_{3}\right)$ & $(1,1)$ & $(3,-2)$ & $(2,-2)$ & $(1,1)$ & $(2,-1)$ & $(2,-2)$ & $(3,-1)$ & $(1,1)$ & $(1,1)$ & $(3,-1)$ & $\ldots$ & $(5,4)$ & $\ldots$ \\
\hline$A_{\mathrm{p}}^{1}$ & 1.94 & 1.29 & 1.94 & 1.29 & 1.94 & 1.29 & 1.60 & 1.60 & 0.91 & 0.91 & $\ldots$ & 1.29 & $\ldots$ \\
\hline$A_{\mathrm{p}}^{2}$ & 1.78 & 1.78 & 1.78 & 1.78 & 1.78 & 1.78 & 3.12 & 3.12 & 3.12 & 3.12 & $\ldots$ & 1.78 & $\ldots$ \\
\hline$A_{\mathrm{p}}^{3}$ & 0.66 & 0.43 & 0.46 & 0.66 & 0.32 & 0.46 & 0.55 & 0.54 & 0.54 & 0.55 & $\ldots$ & 2.22 & $\ldots$ \\
\hline$v_{\mathrm{r}, \max }$ & 1.29 & 0.61 & 1.17 & 0.67 & 1.21 & 0.63 & 0.73 & 0.72 & 0.28 & 0.26 & $\ldots$ & 0.61 & $\ldots$ \\
\hline$v_{\mathrm{t}, \max }$ & 7.28 & 17.37 & 8.84 & 13.83 & 4.75 & 14.6 & 28.7 & 29.1 & 29.5 & 29.1 & $\ldots$ & 51.40 & $\ldots$ \\
\hline$i$ & 55 & 55 & 55 & 55 & 55 & 55 & 85 & 85 & 85 & 85 & $\ldots$ & 55 & $\ldots$ \\
\hline$v_{\Omega}$ & 23 & 12 & 25 & 11 & 19 & 11 & 35 & 35 & 35 & 35 & $\ldots$ & 12 & $\ldots$ \\
\hline$\sigma$ & 17 & 19 & 16 & 19 & 18 & 19 & 11 & 11 & 11 & 11 & $\ldots$ & 7 & $\ldots$ \\
\hline$\Sigma$ & 5.95 & 5.97 & 5.97 & 5.99 & 6.03 & 6.04 & 6.04 & 6.04 & 6.07 & 6.07 & $\ldots$ & 6.14 & $\ldots$ \\
\hline$\left(\ell_{1}, m_{1}\right)$ & $(1,1)$ & $(1,1)$ & $(1,1)$ & $(2,-2)$ & $(2,-2)$ & $(2,-2)$ & $(3,-3)$ & $(1,1)$ & $(1,1)$ & $(2,-2)$ & $\ldots$ & $(2,-2)$ & $\cdots$ \\
\hline$\left(\ell_{2}, m_{2}\right)$ & $(3,-1)$ & $(3,-1)$ & $(3,-1)$ & $(3,-1)$ & $(3,-1)$ & $(3,-1)$ & $(3,-1)$ & $(3,-1)$ & $(2,-1)$ & $(3,-1)$ & $\ldots$ & $(3,-1)$ & $\ldots$ \\
\hline$\left(\ell_{3}^{\prime}, m_{3}^{\prime}\right)$ & $(2,-2)$ & $(2,-1)$ & $(1,1)$ & $(2,-2)$ & $(1,1)$ & $(2,-1)$ & $(5,-3)$ & $(1,0)$ & $(5,3)$ & $(5,-3)$ & $\ldots$ & $(2,0)$ & $\ldots$ \\
\hline$A_{\mathrm{p}}^{1}$ & 1.82 & 1.59 & 1.59 & 0.96 & 0.96 & 0.96 & 1.20 & 1.82 & 1.50 & 0.86 & $\ldots$ & 0.96 & $\ldots$ \\
\hline$A_{\mathrm{p}}^{2}$ & 1.59 & 2.16 & 2.16 & 2.16 & 2.16 & 2.16 & 1.00 & 1.59 & 2.80 & 0.88 & $\ldots$ & 2.16 & $\ldots$ \\
\hline$A_{\mathrm{p}}^{\prime 3}$ & 0.31 & 0.32 & 0.40 & 0.23 & 0.40 & 0.32 & 2.39 & 0.46 & 1.80 & 1.80 & $\ldots$ & 0.39 & $\ldots$ \\
\hline$v_{\mathrm{r}, \max }$ & 0.99 & 1.22 & 1.35 & 0.29 & 0.52 & 0.39 & 0.78 & 1.12 & 0.27 & 0.65 & $\ldots$ & 0.50 & $\ldots$ \\
\hline$v_{\mathrm{t}, \max }$ & 5.44 & 4.23 & 4.55 & 4.53 & 4.48 & 3.58 & 29.0 & 3.62 & 33.1 & 26.4 & $\ldots$ & 4.50 & $\ldots$ \\
\hline$i$ & 55 & 70 & 70 & 70 & 70 & 70 & 80 & 55 & 85 & 85 & $\ldots$ & 70 & $\ldots$ \\
\hline$v_{\Omega}$ & 20 & 27 & 27 & 27 & 31 & 27 & 9 & 20 & 29 & 20 & $\ldots$ & 27 & $\ldots$ \\
\hline$\sigma$ & 18 & 15 & 15 & 15 & 13 & 15 & 3 & 18 & 3 & 12 & $\ldots$ & 15 & $\ldots$ \\
\hline$\Sigma$ & 6.08 & 6.09 & 6.10 & 6.10 & 6.12 & 6.12 & 6.13 & 6.13 & 6.14 & 6.14 & $\ldots$ & 6.17 & $\ldots$ \\
\hline
\end{tabular}

frequency. For each tested $(\ell, m, i)$, the velocity amplitude $A_{\mathrm{p}}$ was chosen so that the theoretical amplitudes of the first moment are equal to the observed ones (see Table 3 ).

The results of the mode identification by means of the nonrotating formalism using $f_{1}, f_{2}$ and $f_{3}$ and using $f_{1}, f_{2}$ and $f_{3}^{\prime}$ are given in respectively the upper and lower part of Table 4. We used $K_{1}=11, K_{2}=12, K_{3}=14$ and $K_{3}^{\prime}=16$. We first of all find that the discriminating function $\Sigma$ has lower values for the combination $f_{1}, f_{2}, f_{3}$, giving slight preference for that combination. A clear conclusion is that none of the three modes is axisymmetric. One can conclude that the choice of the frequency for the third mode does not influence the idenfication of the second mode, for which we systematically find $\left(\ell_{2}, m_{2}\right)=(3,-1)$ or $(2,-1)$. The most likely identification for $f_{1}$ is $\left(\ell_{1}, m_{1}\right)=(1,1)$, although $(3,-2)$ and $(2,-2)$ also occur among the best possibilities. The second solution may then point towards components of a multiplet, as $f_{1}, f_{2}$ are close frequencies. One also remarks that, even if it is difficult to determine the third mode, both identifications do not differ very much.

We then performed a mode identification by means of Lee $\&$ Saio's formalism. The $K$-values were computed using the corotating angular frequency related to the observed one by $\omega_{c}=\omega_{\mathrm{obs}}+m \Omega$, where $\Omega$ is the angular frequency of rotation. We checked each time if the $K$-values remain sufficiently low in order to obtain physically relevant velocity values, i.e. we eliminate too large $K$-values. For computation time reasons, we tested $v_{\Omega}$ from 5 to $35 \mathrm{~km} \mathrm{~s}^{-1}$ with a step $5 \mathrm{~km} \mathrm{~s}^{-1}$. The results using $f_{1}, f_{2}$ and $f_{3}$ and using $f_{1}, f_{2}$ and $f_{3}^{\prime}$ are given in respectively the upper and lower part of Table 5. The identifications for the first and second modes are confirmed and do not change compared to those derived with the neglect of the effect of rotation.

We find systematically a projected rotational velocity above $9 \mathrm{~km} \mathrm{~s}^{-1}$. Assuming that the rotation frequency equals $f_{1}-$ $f_{2}=0.0195 \mathrm{c} \mathrm{d}^{-1}$ leads to an equatorial rotation velocity of only $3.7 \mathrm{~km} \mathrm{~s}^{-1}$. This excludes the possibility that $f_{1}$ and $f_{2}$ correspond to subsequent components of one multiplet.

Given that we cannot discriminate well between $f_{3}, f_{3}^{\prime}$ and other aliases due to bad time sampling, and that a biperiodic solution explains only about $50 \%$ of the standard deviation present in the first moment, we do not attempt line-profile fitting with the best candidate modes found in Table 4 for $f_{1}$ and $f_{2}$. Indeed, we cannot hope to discriminate between such 
Table 5. The ten best solutions of the mode identification by means of Lee \& Saio's formalism through the discriminant $\Sigma$, using the Si II $4130 \AA$ line. The meanings of the symbols are the same as in Table 4. The second-but-last column contains the solution that occurs at position 20 . The upper and lower part correspond respectively to an identification for $f_{1}, f_{2}, f_{3}$ and for $f_{1}, f_{2}, f_{3}^{\prime}$.

\begin{tabular}{|c|c|c|c|c|c|c|c|c|c|c|c|c|c|}
\hline$\left(\ell_{1}, m_{1}\right)$ & $(1,1)$ & $(3,-2)$ & $(1,1)$ & $(1,1)$ & $(3,-2)$ & $(2,-2)$ & $(2,-2)$ & $(2,-2)$ & $(3,-2)$ & $(3,-2)$ & & $(2,-1)$ & \\
\hline$\left(\ell_{2}, m_{2}\right)$ & $(3,-1)$ & $(3,-1)$ & $(3,-1)$ & $(3,-1)$ & $(3,-1)$ & $(3,-1)$ & $(3,-1)$ & $(3,-1)$ & $(3,-1)$ & $(3,-1)$ & $\ldots$ & $(3,-1)$ & $\ldots$ \\
\hline$\left(\ell_{3}, m_{3}\right)$ & $(2,-2)$ & $(1,1)$ & $(1,1)$ & $(2,-1)$ & $(2,-2)$ & $(1,1)$ & $(2,-2)$ & $(2,-1)$ & $(3,-3)$ & $(2,-1)$ & $\ldots$ & $(2,-2)$ & $\ldots$ \\
\hline$A_{\mathrm{p}}^{1}$ & 2.20 & 0.93 & 2.20 & 2.20 & 0.93 & 0.44 & 0.44 & 0.44 & 0.76 & 0.93 & $\ldots$ & 0.42 & $\ldots$ \\
\hline$A_{\mathrm{p}}^{2}$ & 1.11 & 1.47 & 1.11 & 1.11 & 1.47 & 0.74 & 0.74 & 0.74 & 1.29 & 1.47 & $\ldots$ & 0.36 & $\ldots$ \\
\hline$A_{\mathrm{p}}^{3}$ & 0.21 & 0.72 & 0.76 & 0.22 & 0.33 & 0.80 & 0.12 & 0.17 & 0.28 & 0.27 & $\ldots$ & 0.07 & $\ldots$ \\
\hline$v_{\mathrm{r}, \max }$ & 1.09 & 0.58 & 1.22 & 1.10 & 0.51 & 0.23 & 0.14 & 0.11 & 0.45 & 0.50 & $\ldots$ & 0.14 & $\ldots$ \\
\hline$v_{t, \max }$ & 9.22 & 14.86 & 6.42 & 4.93 & 15.91 & 12.68 & 16.88 & 11.53 & 21.48 & 16.43 & $\ldots$ & 12.36 & $\ldots$ \\
\hline$K_{1}$ & 8.47 & 16.33 & 8.47 & 8.47 & 16.33 & 43.54 & 43.54 & 43.54 & 20.04 & 16.33 & $\ldots$ & 23.60 & $\ldots$ \\
\hline$K_{2}$ & 17.32 & 14.32 & 17.32 & 17.32 & 14.32 & 21.37 & 21.37 & 21.37 & 15.71 & 14.32 & $\ldots$ & 25.34 & $\ldots$ \\
\hline$K_{3}$ & 35.16 & 11.99 & 10.22 & 21.29 & 21.30 & 8.81 & 68.44 & 26.90 & 40.78 & 17.26 & $\ldots$ & 137.01 & $\ldots$ \\
\hline$i$ & 55 & 55 & 55 & 55 & 55 & 55 & 55 & 55 & 55 & 55 & $\ldots$ & 50 & $\ldots$ \\
\hline$v_{\Omega}$ & 20 & 10 & 20 & 20 & 10 & 30 & 30 & 30 & 15 & 10 & $\ldots$ & 35 & $\ldots$ \\
\hline$\sigma$ & 18 & 19 & 18 & 18 & 19 & 14 & 14 & 14 & 18 & 19 & $\ldots$ & 12 & $\ldots$ \\
\hline$\Sigma$ & 6.08 & 6.09 & 6.10 & 6.13 & 6.14 & 6.14 & 6.14 & 6.15 & 6.16 & 6.17 & $\ldots$ & 6.21 & \\
\hline$\left(\ell_{1}, m_{1}\right)$ & $(1,1)$ & $(1,1)$ & $(1,1)$ & $(2,-2)$ & $(2,-2)$ & $(2,-2)$ & $(1,1)$ & $(3,-3)$ & $(3,-1)$ & $(3,-3)$ & $\ldots$ & $(3,-3)$ & $\ldots$ \\
\hline$\left(\ell_{2}, m_{2}\right)$ & $(3,-1)$ & $(3,-1)$ & $(3,-1)$ & $(3,-1)$ & $(3,-1)$ & $(3,-1)$ & $(3,-1)$ & $(3,-2)$ & $(2,-2)$ & $(3,-2)$ & $\ldots$ & $(3,-2)$ & $\ldots$ \\
\hline$\left(\ell_{3}^{\prime}, m_{3}^{\prime}\right)$ & $(2,-1)$ & $(2,-2)$ & $(2,1)$ & $(1,0)$ & $(2,-1)$ & $(2,-2)$ & $(1,0)$ & $(2,-2)$ & $(2,-2)$ & $(3,-3)$ & $\ldots$ & $(3,-1)$ & $\ldots$ \\
\hline$A_{\mathrm{p}}^{1}$ & 2.06 & 2.06 & 2.06 & 0.66 & 0.66 & 0.66 & 2.06 & 0.77 & 0.79 & 0.77 & $\ldots$ & 0.77 & $\ldots$ \\
\hline$A_{\mathrm{p}}^{2}$ & 0.99 & 0.99 & 0.99 & 0.99 & 0.99 & 0.99 & 0.99 & 2.35 & 1.15 & 2.35 & $\ldots$ & 2.35 & $\ldots$ \\
\hline$A_{\mathrm{p}}^{\prime 3}$ & 0.14 & 0.13 & 0.28 & 0.46 & 0.14 & 0.13 & 0.46 & 0.16 & 0.45 & 0.17 & $\ldots$ & 0.32 & $\ldots$ \\
\hline$v_{\mathrm{r}, \max }$ & 1.02 & 0.96 & 1.01 & 0.13 & 0.14 & 0.10 & 1.01 & 0.30 & 0.42 & 0.31 & $\ldots$ & 0.17 & $\ldots$ \\
\hline$v_{\mathrm{t}, \max }$ & 3.07 & 5.70 & 5.66 & 8.18 & 8.09 & 12.35 & 3.34 & 40.11 & 29.30 & 40.31 & $\ldots$ & 39.32 & $\ldots$ \\
\hline$K_{1}$ & 8.47 & 8.47 & 8.47 & 25.15 & 25.15 & 25.15 & 8.47 & 17.93 & 15.24 & 17.93 & $\ldots$ & 17.93 & 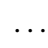 \\
\hline$K_{2}$ & 17.32 & 17.32 & 17.32 & 17.32 & 17.32 & 17.32 & 17.32 & 16.16 & 22.77 & 16.16 & $\ldots$ & 16.16 & $\ldots$ \\
\hline$K_{3}^{\prime}$ & 25.11 & 43.57 & 11.44 & 16.31 & 25.11 & 43.57 & 16.31 & 23.10 & 35.08 & 28.21 & $\ldots$ & 19.26 & $\ldots$ \\
\hline$i$ & 55 & 55 & 55 & 55 & 55 & 55 & 55 & 85 & 30 & 85 & $\ldots$ & 85 & $\ldots$ \\
\hline$v_{\Omega}$ & 20 & 20 & 20 & 20 & 20 & 20 & 20 & 10 & 10 & 10 & $\ldots$ & 10 & $\ldots$ \\
\hline$\sigma$ & 18 & 18 & 18 & 18 & 18 & 18 & 18 & 17 & 20 & 17 & $\ldots$ & 17 & $\ldots$ \\
\hline$\Sigma$ & 6.25 & 6.26 & 6.27 & 6.27 & 6.27 & 6.27 & 6.28 & 6.29 & 6.29 & 6.29 & $\ldots$ & 6.32 & $\ldots$ \\
\hline
\end{tabular}

fits in a meaningful way, as there is clearly still variability due to at least one, and probably even more, low-amplitude modes. Such modes are sort of filtered out in the moment variations but not in the line profiles themselves. For this reason, we have determined theoretical values for $\langle v\rangle,\left\langle v^{2}\right\rangle$ and $\left\langle v^{3}\right\rangle$ for the best solutions listed in the upper panel of Table 4 . For four of these solutions, we compare the moment values with the observed ones in Fig. 6. The four solutions are indicated in italic in Table 4. We point out that all first 15 best solutions result in very similar moment values and that we are unable to discriminate between the different possibilities for the mode identification. The only result that we can conclude upon with certainty is that we are dealing with non-axisymmetric $\ell \leq 3$ modes. Additional data with full coverage of the overall beat-period is needed to obtain unique mode identifications. A visual check shows that the $(\ell, m)$ combinations from position 16 onwards explain less well the observed moment variations. For comparison, we also list in Table 4, and show in Fig. 6, the solution that occurs on position 20. One can see from the dashed line in the lowest and rightmost panel of Fig. 6 that this solution leads to a too large amplitude for $f_{3}$ in the third moment. Moreover, its maximal tangential velocity is quite high. We conclude that we cannot discriminate between some 15-20 solutions from the discriminant. It will become possible to obtain unambiguous mode identifications for this star if we are able to detect a limited number of additional modes, by combining the results of the discriminant and of seismic models (see further below).

For all solutions $i \in\left[50^{\circ}, 85^{\circ}\right], v_{\Omega} \in[10,35] \mathrm{km} \mathrm{s}^{-1}$. For the radius $3.7 R_{\odot}$, this leads to a rotation period between 4.3 and 18.7 days.

\section{Comparison with theoretical pulsation models}

The mode identification for $f_{1}$ and $f_{2}$ is the first one derived from a spectroscopic time series for this SPB. Moreover, we find evidence for an $\ell=3$ mode, which is seldom observed 

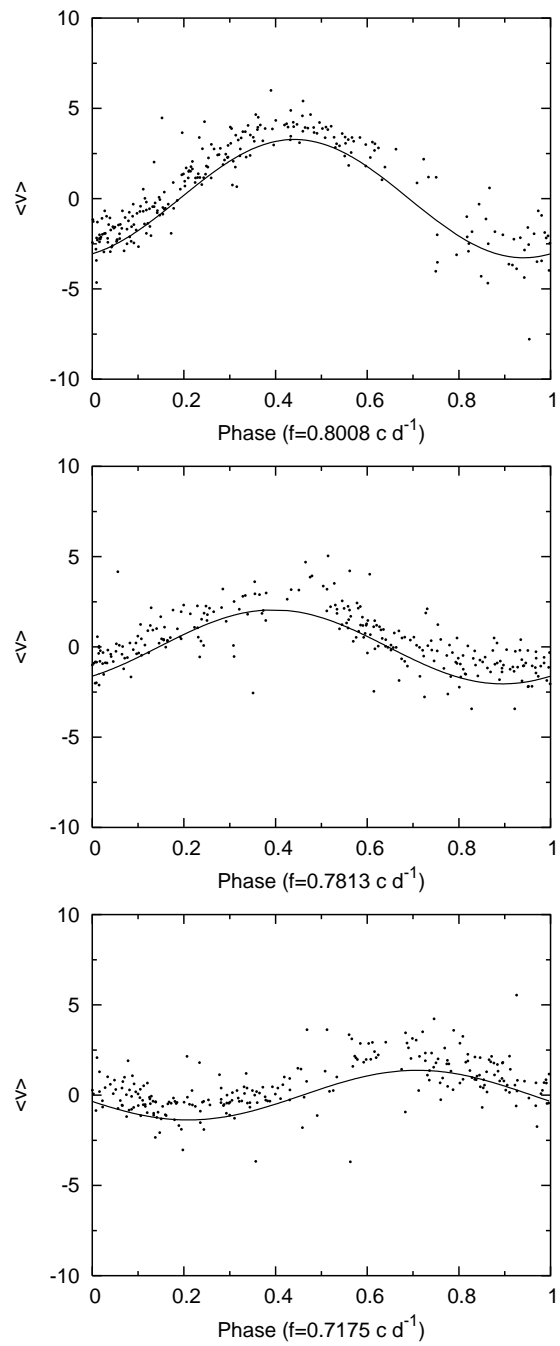
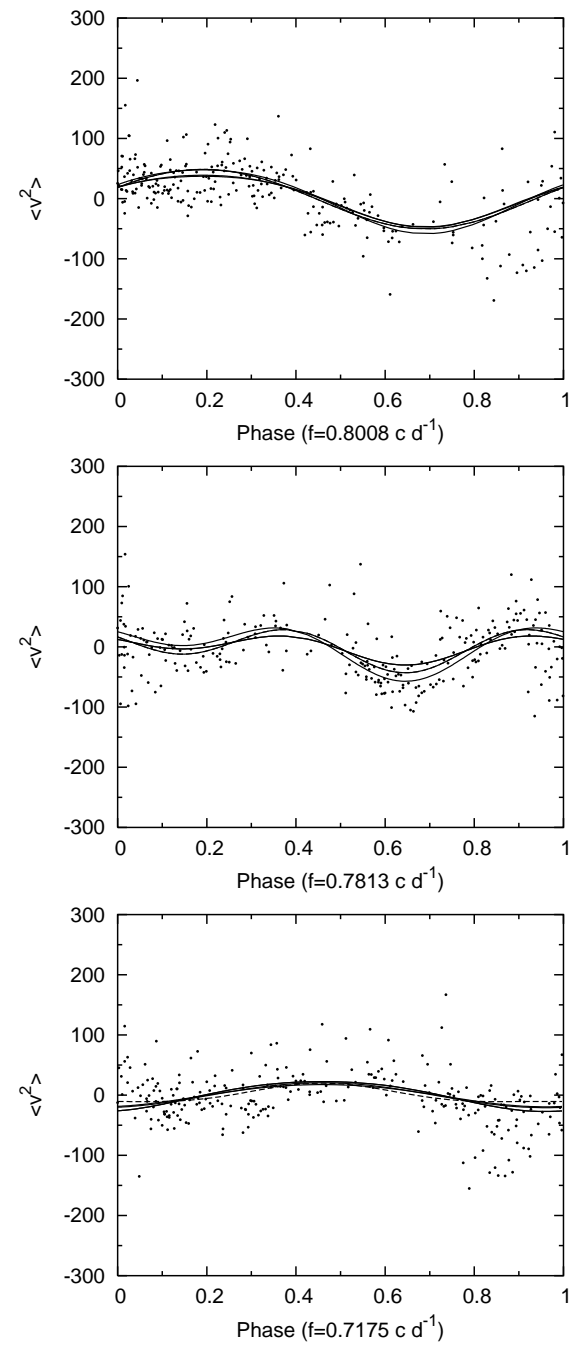
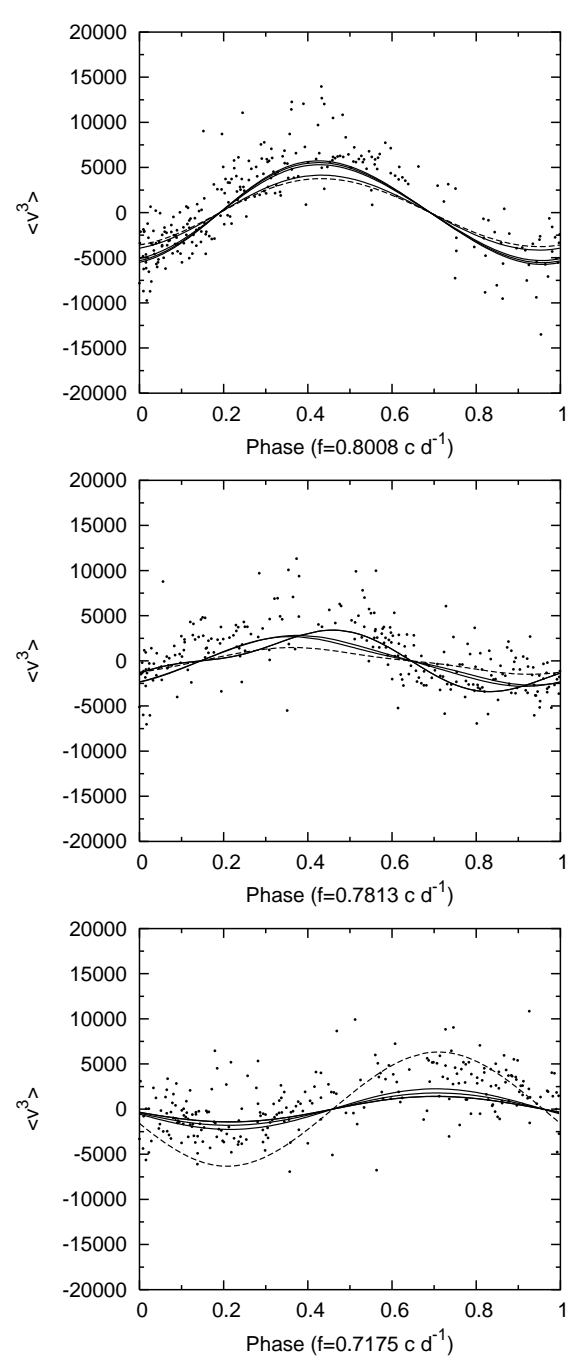

Fig. 6. Comparisons between the observed first three moments (dots) to four of the best solutions (full lines) given in italic in Table 4 . The dashed line represents the solution that occurs at position 20 in Table 4. Left: from top to bottom: phase diagram for $f_{1}$ of $\langle v>$ prewhitened with $f_{2}$ and $f_{3}$, phase diagram for $f_{2}$ of $<v>$ prewhitened with $f_{1}$ and $f_{3}$, phase diagram for $f_{3}$ of $<v>$ prewhitened with $f_{1}$ and $f_{2}$. Middle: from top to bottom: phase diagram for $f_{1}$ of $<v^{2}>$ prewhitened with all frequencies except $f_{1}$ and $2 f_{1}$, phase diagram for $f_{2}$ of $<v^{2}>$ prewhitened with all frequencies except $f_{2}$ and $2 f_{2}$, phase diagram for $f_{3}$ of $<v^{2}>$ prewhitened with all frequencies except $f_{3}$ and $2 f_{3}$. Right: from top to bottom: phase diagram for $f_{1}$ of $<v^{3}>$ prewhitened with all frequencies except $f_{1}, 2 f_{1}$ and $3 f_{1}$, phase diagram for $f_{2}$ of $<v^{3}>$ prewhitened with all frequencies except $f_{2}, 2 f_{2}$ and $3 f_{2}$, phase diagram for $f_{3}$ of $<v^{3}>$ prewhitened with all frequencies except $f_{3}, 2 f_{3}$ and $3 f_{3}$. Note that the errors on $\langle v\rangle,\left\langle v^{2}\right\rangle$ and $\left\langle v^{3}\right\rangle$ are respectively given by $0.45 \mathrm{~km} \mathrm{~s}^{-1}, 20\left(\mathrm{~km} \mathrm{~s}^{-1}\right)^{2}$ and $1000\left(\mathrm{~km} \mathrm{~s}^{-1}\right)^{3}$.

in pulsating stars. In order to check if such a solution is compatible with theoretical model predictions, we have determined evolutionary model sequences from the main sequence that pass the position of HD 147394 in the HR diagram (see Fig. 7) with the Code Liégeois d'Évolution Stellaire written by one of us (RS), assuming no convective overshooting. For each model with $4.15 \leq \log T_{\text {eff }} \leq 4.19$ we have subsequently calculated the oscillation frequencies using a standard adiabatic code (Boury et al. 1975). For each evolutionary sequence, we have selected the models which give an exact fit for $f_{1}$ and $f_{2}$ according to the identification $\left(\ell_{1}, m_{1}\right)=(1,1)$ and $\left(\ell_{2}, m_{2}\right)=$ $(3,-1)$, taking into account the Ledoux rotational splitting constant and by varying the equatorial rotation velocity in the range $[10,50] \mathrm{km} \mathrm{s}^{-1}$ with a step of $0.1 \mathrm{~km} \mathrm{~s}^{-1}$. Figure 8 represents theoretical frequencies compared to observed ones for one of the many models that we found compatible with the observations. For this example, $f_{1}$ corresponds to the $g_{12}$ mode and $f_{2}$ to the $g_{40}$ mode. We note that, even with a constraint coming from mode identification, the sets of theoretical frequencies are quite dense, which makes it difficult to find one unique solution, especially as the rotation frequency is also a free parameter to a certain extend.

We have limited ourselves to the combination $\left(\ell_{1}, m_{1}\right)=$ $(1,1)$ and $\left(\ell_{2}, m_{2}\right)=(3,-1)$ for our compatibility check with the models. We stress, however, that other mode identifications would also lead to agreement with models of slightly different stellar parameters.

\section{Summary}

Our study of the slowly pulsating B star HD 147394 was based on 250 high-resolution high signal-to-noise spectra spread 


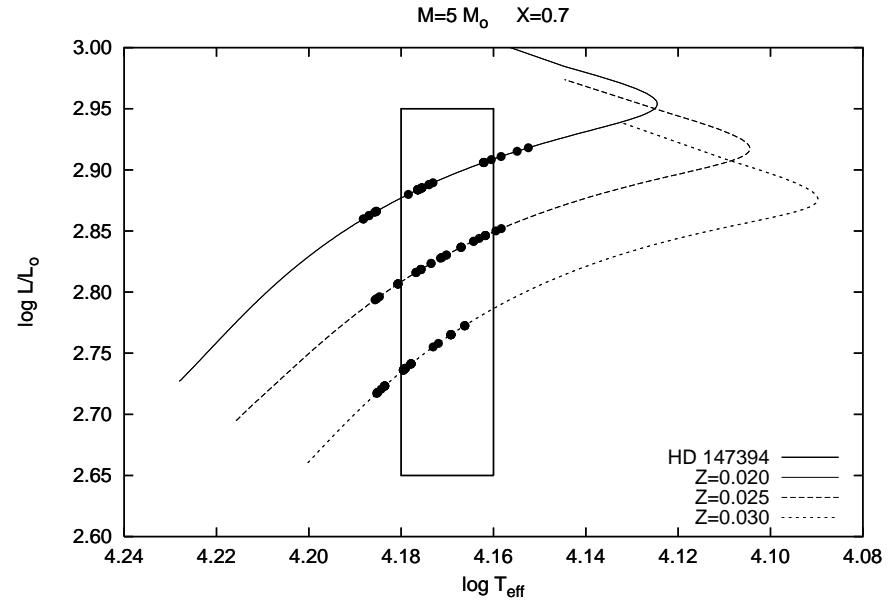

Fig. 7. Evolutionary tracks of a $5 M_{\odot}$ star without overshooting, with $X=0.70$ and for different values of the metallicity $Z$, computed with CLES. The box delimits the position of HD 147394 in the HR diagram derived from photometry. Dots represent models that fit $f_{1}$ and $f_{2}$ according to the mode identification $\left(\ell_{1}, m_{1}\right)=(1,1)$ and $\left(\ell_{2}, m_{2}\right)=(3,-1)$.

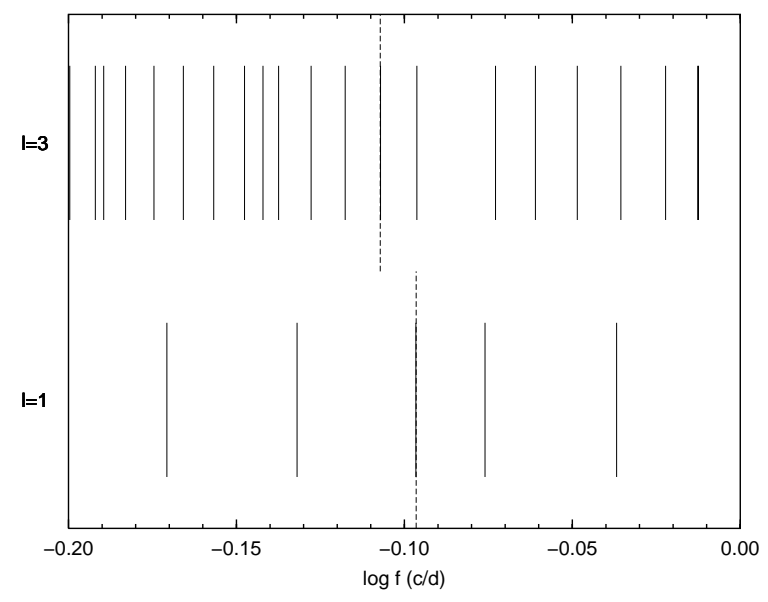

Fig. 8. Theoretical frequencies for one of the models that fit observed frequencies (dashed lines). The $\ell=2$ modes are not shown, but have a density in between the $\ell=1$ and $\ell=3$ modes.

over 460 days. The moment variations of the Si II 4128-4130 doublet clearly reveal multiperiodicity with frequencies $f_{1}=$ $0.8008 \mathrm{~cd}^{-1}, f_{2}=0.7813 \mathrm{~cd}^{-1}$ and $f_{3}=0.7175 \mathrm{~cd}^{-1}$ or its aliases due to the time sampling. We performed a mode identification by means of a new version of the moment method which identifies multiple modes simultaneously, leading to only one derived estimate for $v_{\Omega}, i$ and $\sigma$ for each of the $(\ell, m)$ combinations. We did it by using the non-rotating formalism as well as the one derived by Lee \& Saio (1987) for low-frequency gmode pulsators. Both identifications lead to almost the same list of best candidate solutions whatever is the chosen value for the third frequency. The identification for the modes is not unique but we conclude that they are non-axisymmetric with $\ell \leq 3$. The rotation period of the star must be between 4 and 19 days.

It turns out that less than 20 combinations of $(\ell, m)$ are found to be equivalent by the discriminant for this $\mathrm{SPB}$. It therefore should be possible to derive correct mode identifications from the discriminant and seismic modelling should we detect a small additional number of modes in the star.

Despite the fact that HD 147394 is currently the SPB for which we have the most high-quality spectra available, its frequency spectrum revealed only three modes so far due to severe alias problems in the dataset. In view of the dense frequency spectra of potential modes (see Fig. 8), it is at present not the best target for a seismic analysis. Indeed, in De Cat \& Aerts (2002) we find several SPBs with a larger number of detected modes, mainly in those objects for which very long-term multicolour photometry is available. It is our intention to apply the moment method by Briquet \& Aerts (2003) and the photometric amplitudes method by Dupret et al. (2003) to all the targets in the list of De Cat \& Aerts (2002). With state-of-the-art mode identifications at hand, we subsequently plan to compute numerous theoretical models to derive the stellar parameters of the SPBs with unprecedented precision and, if possible, to derive information on the (internal) rotational behaviour of these massive gravity-mode oscillators, according to the strategy outlined in Aerts et al. (2003).

Acknowledgements. We thank the referee for very constructive comments which helped to improve our manuscript. This work was supported by the P.A.I. (Pôle d'Attraction Interuniversitaire) and was carried out within the Belgian Asteroseismology Group.

\section{References}

Aerts, C., De Pauw, M., \& Waelkens, C. 1992, A\&A, 266, 294

Aerts, C. 1996, A\&A, 314, 115

Aerts, C., De Cat, P., Peeters, E., et al. 1999, A\&A, 343, 872

Aerts, C., Scuflaire, R., \& Thoul, A. 2003, in IAU Symp. 215, Stellar Rotation, ed. A. Maeder, \& P. Eenens, ASP Conf. Ser., in press

Boury, A., Gabriel, M., Noels, A., Scuflaire, R., \& Ledoux, P. 1975, A\&A, 41, 279

Breger, M., Stich, J., Garrido, R., et al. 1993, A\&A, 271, 482

Briquet, M., \& Aerts, C. 2003, A\&A, 398, 687

De Cat, P., Aerts, C., De Ridder, J., et al. 2000, A\&A, 355, 1015

De Cat, P. 2001, Ph.D. Thesis, Katholieke Universiteit Leuven, Belgium

De Cat, P. 2002, in IAU Colloq. 185: Radial and Nonradial Pulsations as Probes of Stellar Physics, ed. C. Aerts, T. R. Bedding, \& J. Christensen-Dalsgaard, ASP Conf. Ser., 259, 196

De Cat, P., \& Aerts, C. 2002, A\&A, 393, 965

Dupret, M.-A., De Ridder, J., De Cat, P., et al. 2003, A\&A, 398, 677

Flower, P. J. 1996, ApJ, 469, 355

Künzli, M., North, P., Kurucz, R. L., \& Nicolet, B. 1997, A\&AS, 122, 51

Lee, U., \& Saio, H. 1987, MNRAS, 224, 513

Masuda, S., \& Hirata, R. 2000, A\&A, 356, 209

Mathias, P., Aerts, C., Briquet, M., et al. 2001, A\&A, 379, 905

Pamyatnykh, A. A. 1999, Acta Astron., 49, 119

Roberts, D. H., Lehár, J., \& Dreher, J. W. 1987, AJ, 93, 968

Scargle, J. D. 1981, ApJS, 45, 1

Schaller, G., Schaerer, D., Meynet, G., \& Maeder, A. 1992, A\&AS, 96, 269

Smith, K. C., \& Dworetsky, M. M. 1993, A\&A, 274, 335

Smith, K. C. 1997, A\&A, 319, 928

Stellingwerf, R. F. 1978, ApJ, 224, 953

Wade, R. A., \& Rucinski, S. M. 1985, A\&AS, 60, 471

Waelkens, C. 1991, A\&A, 244, 107

Waelkens, C., Aerts, C., Kestens, E., et al. 1998, A\&A, 330, 215 